\title{
The design of energy storage welding machine with high voltage based on the PIC single chip
}

\author{
Rongsheng $L v^{1,}$ a , Rui Yang ${ }^{2, b}$ \\ 1School of Management, Tianjin University of Technology, Tianjin, China \\ 2School of Management, Tianjin University of Technology, Tianjin, China \\ a943299063@qq.com, byangrui19880703@126.com
}

Keywords: stored-energy welding, PIC18F4520 SCM, the peripheral interface technology of SCM

\begin{abstract}
This paper discussed the design of the energy storage welding machine which was controlled by PIC18f4520 SCM, energy storage welding machine is a kind of resistance welding. The working principle of the resistance welding uses the electrode press the weld port and power on it. The contact resistance of the weld port between the electrode produce joule heat can melt the metal to weld. Stored-energy welding machine is also called the capacitor stored energy welding machine, it's working principle is using the capacitor to store energy, once the energy is enough to melt the metal, capacitor discharges. Compared with other welding method, energy storage welding machine has the lower Instantaneous power, balanced load of each phase and high power factor . the energy storage welding machine is used to weld the welding of nonferrous metals commonly.As the energy storage welding machine's charging voltage value is controlled by the method of the Voltage comparison trigger, it control precision is low, and the cost is higher. The purpose of this design is reconstructing of the existing structure of stored energy welding machine and the use the PIC18f4520 MCU constitute a control system to achieve precise control.
\end{abstract}

\section{Introduction}

AStored energy welding machine is also called the capacitor stored energy welding machine.The main working principle is to electrical energy stored in the capacitor, when the energy reaches the welding conditions (i.e., energy is enough to make a small area of solder melts), capacitance discharge moment for welding. Stored energy welding machine is widely used in low carbon steel, stainless steel, copper, alloy and other metal welding.Compared with other methods stored energy welding machine smaller impact on power grid, and from the grid access instantaneous power is low, high power factor, output energy concentration.

\section{The structure of the stored energy welding machine}

As shown in the figure below, the working principle of stored energy welding. When $\mathrm{S}$ accesses $\mathrm{S} 1$, capacitance $\mathrm{C}$ is charged by the power $\mathrm{U}$. Once the voltagevalue of capacitance $\mathrm{C}$ up to the setting voltage value, and $\mathrm{S}$ accesses $\mathrm{S} 2$,Capacitance $\mathrm{C}$ is discharged through the primary winding of transformer T1, At the same time the secondary winding of transformer T1 induce current and Access to the welding circuit. The resistance $\mathrm{R}$ can control the charging current and charging time. Because the current in the welding circuitis large, the instantaneous heat can be used for welding.

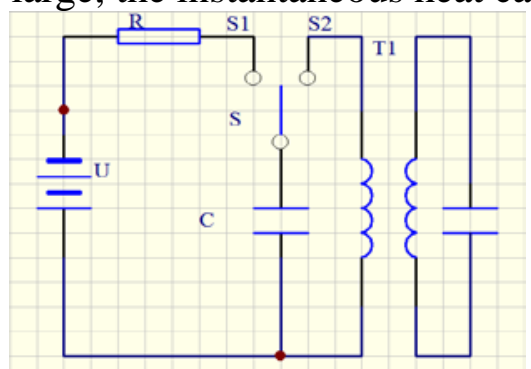

Figure1 The working principle of stored energy welding 
The working principle of stored energy welding is to control the capacitor charging and discharging. The key ofthestored-energywelding isthedesign of capacitorcharginganddischarging.

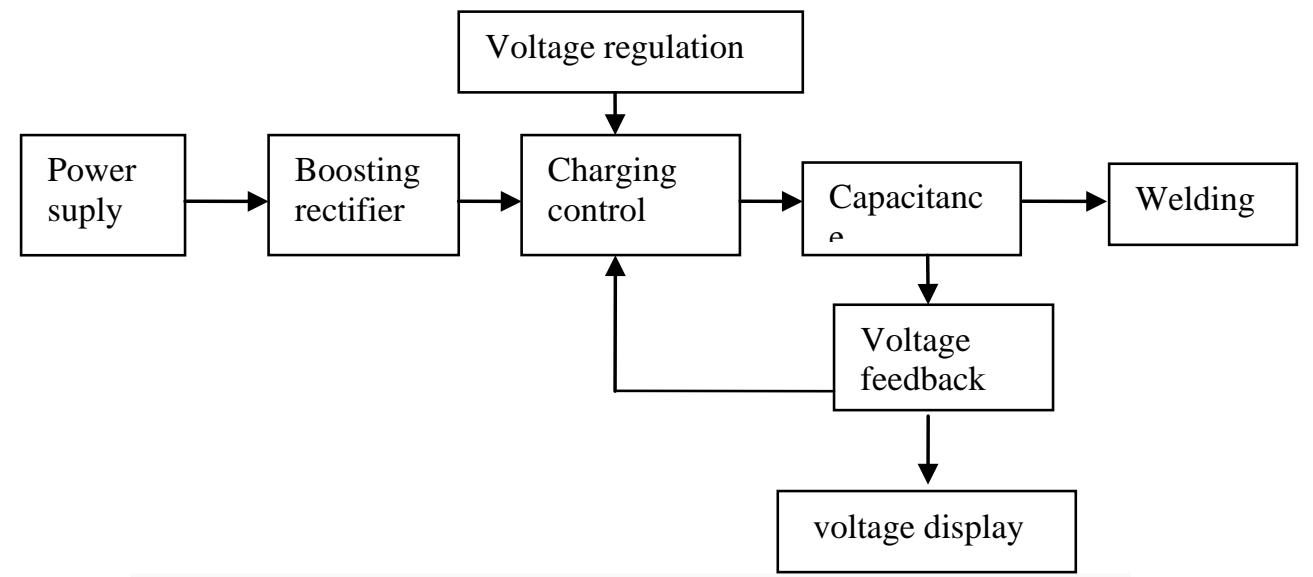

Figure2The structure of the stored energy welding machine

The overall structure diagram above is the stored-energy welding machine. The power part access to 380VIndustrial power. After boosting rectifier, toobtain a high voltage dc power supply of constant voltage. By designing the PIC18f4520 MCU peripheral interface and program to control the capacitor charge/discharge, at the same time on the outside of the equipment design show/adjustment module implemented the adjustment of the stored energy-welding machine monitoring. Based on the voltage value samples at the ports of the capacitance, MCU system to adjust the charging control part guarantee on both ends of the capacitor voltage constant to ensure welding quality, thus forming a closed loop control system.

\section{The design of periphery of the stored-energy welding machine main circuit}

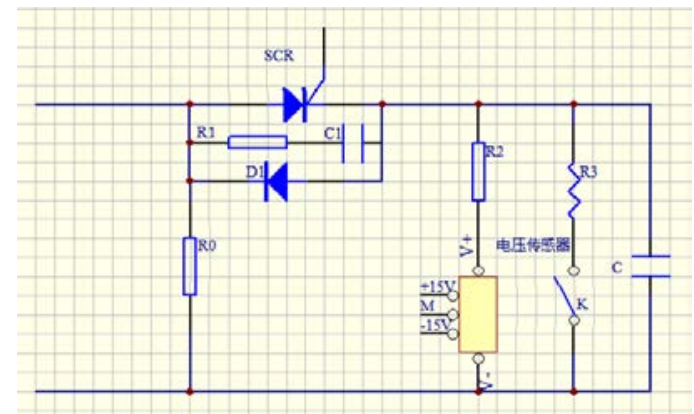

Figure3Charging control circuit

The above is the stored-energy welding machine recharge control circuit. Through the boosting rectifier,current through SCR control after charging, when the SCR is gating, current flows into the capacitor C.AS the voltage at the ports of the capacitor has a high voltage, after the capacitor charging, if suddenly the power boost rectifier part switch offmakesthe lower voltage,the capacitance will produce high surge voltage at the ports. So using of resistor R1 and capacitor C1 composed of resistive capacitive absorbing circuit in order to absorb the surge voltage and protect the SCR.D1 as a Fly-wheel diode has the same effect.

In order to achieve the capacitance $\mathrm{C}$ on both ports of the voltage control, put the voltage sensor access on both ports of the capacitor ,voltage sensor will convert $0 \sim 1600 \mathrm{~V}$ high voltage to $0 \sim 15 \mathrm{~V}$ low voltage (NET_M) then transfer the voltage value to MCU.The program can show/modulate the capacitance voltage on both ports.

R3is the electric stove wire that use for consuming the energy that stored in the Capacitance. When needs to discharge,the charging circuit disconnects, and K turns closed. The energy that stored in the Capacitanceconsume in R3, to ensure the safety of the consumer, and protect the element in the stored energy welding machine. 


\section{The design of SCM Control system}

The choice of MCU. SCM system is the core part of the stored-energy welding machine. After the selection, the PIC18f4520 chip is elected.

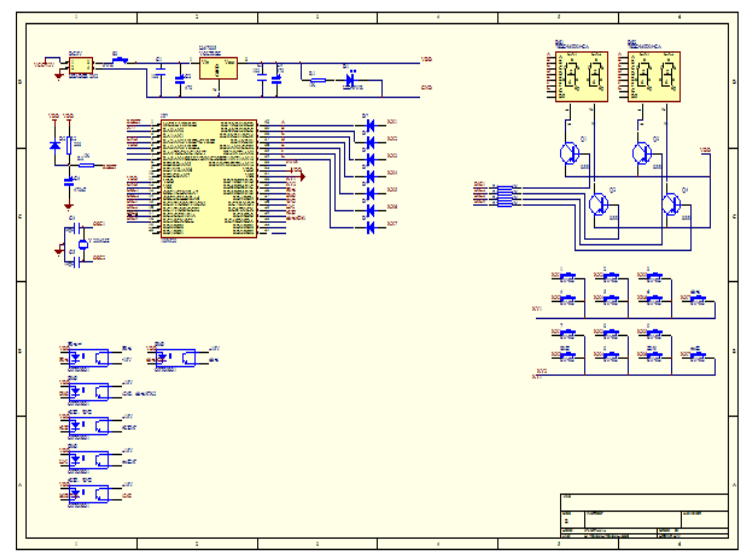

Figure4The SCM Control system

The Power supply. LM7805 is used forProviding $5 \mathrm{v}$ voltage . It requires that the input voltage is greater than two times the output voltageand less than three times the output voltage due to the requirement of heat dissipation.So choose the $12 \mathrm{v}$ switching mode power supply as a input. For reasons of stability ,place the filter capacitor at the input and output ports.

MCU reset circuit. PIN_1is a programming-voltage/reset/digital-input reuse port. When it is configured as a reset port, the MCU will be restarted by a low levelsignal, so in order to make the MCU run ,the PIN_1 should stay in a high level.When the S0 is closed, the capacitive will be charged Slowly and prevent voltage jump, D2 will prevent reverse current to protect the MCU.

Crystals. The PIC18F2420/2520/4420/4520 chip can work under ten different oscillation modes. through programming configuration bits in the configuration register $1 \mathrm{H}$ FOSC3: FOSC0, The user can choose the mode of HS.Crystal vibration signal access the port OSC1 and OSC2.

The displays of LED segment. The LED Segment which is selected models for 7SEG-MPX4-CA as a common-anode LED ,is driven by a transistor driver, with a $1 \mathrm{~K}$ resistor to limit the Current value.

Keyboard.In order to save the MCU I/O port , the keyboard module and the LED segment displaysis accessed to the same I/O port. When LED segment displays is running, the current is controlled by MCU to flow to LED segment displays, as the line scan signal of the keyboard module is disabled, the LED segment displays will not mess. And in the same way, when the keyboard module is running,the selected signal of the LED segment displays is closed, so the LED segment displays is also not mess. As the SCM refresh the LED segment displays and the keyboard moduleat breakneck speed. Due to the nature of the persistence of vision ,the flashing of the LED segment displaysnot be detected, ensure the LED segment displays and keyboard module running in the "same time".

External interface. First, charging control part: SCM Control the thyristor driving circuit by the Optocoupler to charging,as the same time, the charging indicatoris lit up, indicate charging. Second, discharge control part: When the voltage value on both ports of the capacitorup to the settingvoltage value, SCM started waiting for orders and Dischargeindicatoris lit up, Once the Discharge key was closed, the MCU control the machine discharge. Third, foot switch: When the voltage value on both ports of the capacitorup to the settingvoltage value, the MCU waiting the orders, any time Stepped on the foot switch, the MCU control the machine Start the welding. Fourth, pressureindicator L3: when the Pressurebutton is Pressed, Pressureindicator is lit up and thebetween solenoidvalve wich control of copper pol falls ,useing pressure switch, can be adjusted the pressure between the copper pol. Fifth, alarmindicato: when the machine has failure,MCU into the process of alarm, the buzzer ring out to Alarm. To achieve the control function of the before mentioned through controlling the peripheral chips and modules . 


\section{Summary}

The stored-energy welding machine is a kind of industry commonly used welding equipment. The design on the stored-energy welding machine was improved by using the professional knowledge learned in the university. For reasons of safety and convenience, PIC Family MCU is selected. It has high stability, simple programming, program module. Of course, for SCM, the working current of stored-energy welding machine is too high will still is the biggest problems of the stability, So at the end of the electrical design, it is necessary to improve the stability of the SCM, such asusing which has the function of electromagnetic shielding case, using screening wire, etc.

Although that the SCM has the characteristic of susceptible to interference, due to its high degree of integration,using SCM system is still a way to save cost and improve product qualityin industrial production.

\section{References}

[1] Salsbury TI.A temperature controller for VAV air-handling untis based on simplified physical models [J].HVAC\&R Research,1998,4 (3) :265-279.

[2] Maidi A, Diaf M.Optimal linear PI fuzzy controller design of a heat exchanger[J]. Chemical Engineering and Processing,2008(47):938-945.

Reference to a chapter in an edited book:

[3] Wang SW. Dynamic simulation of building VAV air-conditioning system and evaluation of EMCS on-line control strategies [J]. Building and Environment, 1999,34(6):681-705.

[4]The chip information of PIC18F4520.[EB/OL] http://www.atmel.com

[5]The chip information of PIC16F873.[EB/OL] http://www.intersil.com

[6]Xun Li. The technology application of AVR single-chip [M]. Beijing University of Aeronautics and Astronautics, 2002.

[7] $\mathrm{Xu} \mathrm{XH,} \mathrm{Wang} \mathrm{SW,} \mathrm{Shi} \mathrm{WZ.} \mathrm{A} \mathrm{robust} \mathrm{sequencing} \mathrm{control} \mathrm{strategy} \mathrm{for} \mathrm{air-handling} \mathrm{units} \mathrm{[J].}$ Building Services Engineering Research and Technology, 2004,25(2):141-158. 